\title{
Functional relationships between myotatic reflex arcs of the lower limb in man: investigation by excitability curves
}

\author{
P. J. DE L W A I DE, M. COR D O N IER, A N D M. CHARLIER \\ From the Section of Neurology and Clinical Neurophysiology, \\ Department of Internal Medicine, University of Liege, Belgium
}

SYNOPSIS In 30 normal subjects, the influence of the reflex activation of one myotatic reflex arc on the excitability of other myotatic reflex arcs of the lower limb has been investigated using excitability curves. Soleus, quadriceps, and short biceps tendon reflexes as well as $\mathrm{H}$ reflex at two different intensities (liminal and $\mathrm{H} \max / 2$ ) were used either as conditioning or as conditioned responses. The reflex activation of the soleus muscle has opposite effects on antagonistic muscle groups of the thigh: facilitation of the quadriceps myotatic arc and inhibition of the short biceps myotatic reflex arc. Conversely, activation of both quadriceps and short biceps muscles leads to a marked and long lasting $( \pm 5000 \mathrm{~ms})$ inhibition of the soleus myotatic reflex arc. The differences of functional organization between proximal and distal myotatic reflex arcs are emphasized and the role of the afferent impulses secondary to the conditioning muscular contraction is discussed.

In normal man, muscular contractions of the lower limb are well coordinated in activities such as walking.

Within the central nervous system, this coordination is achieved by several neuronal networks. The cerebellum plays a determining role in this function but even at the spinal level reciprocal influences between motor nuclei are operating (Lloyd, 1944).

The proprioceptive and exteroceptive peripheral afferent nerves influence this coordination at different levels in the nervous system.

In this study, we have investigated the influence of the reflex activation of one myotatic reflex arc of the lower limb on the excitability of other myotatic arcs. We used the method of excitability curves described by Magladery et al. (1951) and Paillard (1955). These curves, performed with experimental conditions excluding any voluntary contraction, indicate how proprioceptive afferent fibres can modify the excitability of agonist or antagonist myotatic arcs.

Three myotatic reflex arcs of lower limb have been explored: those of soleus, quadriceps, and short biceps muscles.

\section{METHODS}

The investigations were carried out on 30 normal subjects of both sexes; they were paid volunteers, mostly students.

The subject sat in a modified dental chair with the back slightly reclining. The leg made an angle of $120^{\circ}$ with the thigh; the angle between the leg and foot was approximately $110^{\circ}$.

The lower limb was fixed at the foot and at the thigh so that the muscular reflex contractions were isometric.

During the whole experiment, the subject was immobile, with all muscles relaxed, in order to avoid the influence of distant contractions (Jendrassik effect).

Three myotatic reflex arcs of the lower limb were investigated by eliciting their monosynaptic 
reflex: the soleus, quadriceps, and short biceps myotatic reflex arcs.

For the soleus and quadriceps myotatic reflex arcs, cathodal stimulation of their nerves was used: medial popliteal nerve in the popliteal fossa (Hoffmann reflex) and femoral nerve. The advantage of the electrical technique is that it enables the stimulus intensity to be modified. Furthermore, the stimulation does not spread to other muscle groups. We selected two intensities so that the muscular response derived on the oscilloscope was more or less $1 / 20$ th of $\mathrm{H} \max (=\mathrm{H}$ liminal) in the first case and $\mathrm{H} \max / 2$ in the second case. For the Hoffmann reflex we used the methodology described by Desmedt (1973).

The soleus tendon reflex (T Sol) was elicited by an electromagnetic hammer as previously described (Delwaide et al., 1970). The quadriceps (TQ) and short biceps (TSBi) reflexes were elicited by percussing the corresponding tendons with Racia electromagnetic hammers. These hammers deliver, always using the same angle, percussions which are identical in duration and intensity. These hammers were rigidly fixed in front of the tendons in such a way as to evoke a maximal response.

Three pairs of cutaneous electrodes were placed over the three explored muscles (the indifferent electrode being on the tendon); they recorded the electrical activity corresponding to the three monosynaptic reflexes under study. The potentials were amplified by a Tektronix 9A9 plug in amplifier and visualized on a Tektronix 565 oscilloscope; the peak to peak measurement was obtained by a Tektronix digital unit type 280 and printed by a 5050B digital recorder (HewlettPackard).

The changes in the excitability of one myotatic reflex arc after the activation of one of the other two arcs were explored by measuring the amplitude of the monosynaptic response.

The first evoked reflex is the conditioning reflex; the second one is called the test reflex; the second stimulus was applied at progressively increasing intervals after the conditioning stimulus.

The interval of 0 to $5000 \mathrm{~ms}$ after the conditioning response was investigated. 'Point zero' corresponds to the moment in time when there is coincidence between the electrical responses evoked by both conditioning and test stimuli.

During each experiment the values of the non-conditioned reflex were used as references. These values were the mean values of at least five measurements made alternately with the same number of conditioned responses for each inter- val. They were plotted on a graph where the abscissa represented the intervals in ms between the two stimuli, and the ordinate the percentage of control values of reflex amplitudes. There was an interval of at least seven seconds between successive sets of paired reflexes.

\section{RESULTS}

The different possible combinations between three myotatic reflex arcs could potentially be represented by six excitability curves. In fact, the number of combinations is greater because using the $\mathrm{H}$ reflex made it possible to select several critical values: in our experiments, $\mathrm{H}$ liminal and $\mathrm{H} \max / 2$.

CONDITIONING OF PROXIMAL MYOTATIC REFLEX ARC BY ACTIVATION OF DISTAL MYOTATIC REFLEX ARC Conditioning of quadriceps myotatic reflex arc by activation of soleus myotatic reflex arc The activation of the soleus myotatic reflex arc by an electrical stimulation applied with the technique for the $\mathrm{H}$ reflex alters the excitability of the quadriceps reflex arc; this is clearly shown by the method of excitability curves.

Figure IA shows the mean excitability curve (plain line) obtained from seven experiments. In this case, the $\mathrm{H}$ reflex is used as conditioning reflex with a liminal intensity. Each point of this curve corresponds to the mean of at least 35 measurements.

After an inhibitory phase of about $40 \mathrm{~ms}$, there is a rapidly increasing facilitation of the quadriceps tendon reflex (TQ) reaching $100 \%$ of the reference values at the interval of 100 ms. It levels out at this value for more or less $50 \mathrm{~ms}$, then smoothly decreases to reach the reference values after $600 \mathrm{~ms}$.

The standard deviations are represented as two dotted lines on either side of the excitability curve: from $300 \mathrm{~ms}$ onwards they fluctuate between 20 and 35; for intervals of 60 to $300 \mathrm{~ms}$ they are much greater $(\sigma=60$ to 100).

Figure IB shows the excitability curve obtained when the conditioning reflex is equal to $\mathrm{H} \max / 2$. Contrary to the effects of the smaller stimulus, a distinct contraction of the soleus muscle occurs which is clinically detect- 


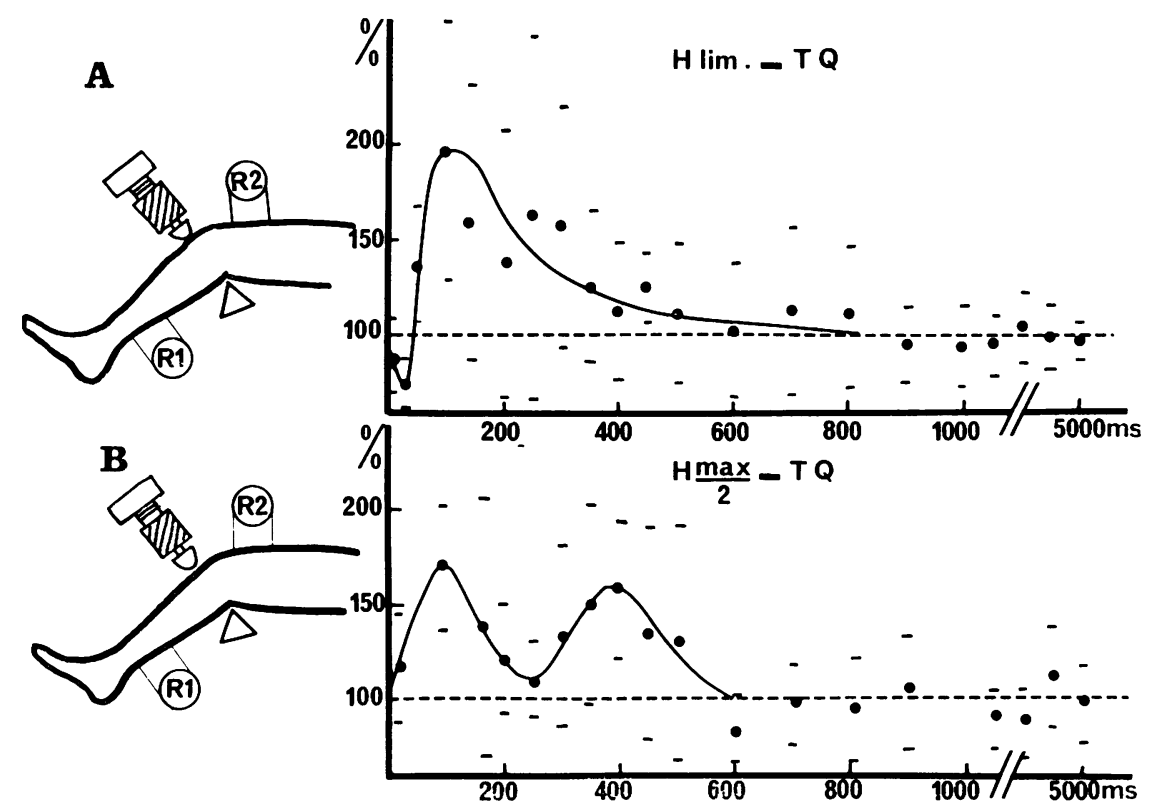

FIG. 1 Left: diagram of the experimental conditions: hammer percussing the tendon; $R$ : recording electrodes; triangle: stimulating electrode in the popliteal fossa. White symbols and number 1: the conditioning reflex. Hatched symbols and number 2: the conditioned response. Right: excitability curves of the quadriceps myotatic reflex arc tested by the tendon reflex after conditioning by $H$ reflex. The ordinate shows the amplitude of the test reflex expressed as a percentage of the control amplitude. The abscissa shows the time interval in $m s$ between the conditioning and the test responses. In $A$, the conditioning $H$ reflex is at liminal intensity. In $B$, the conditioning $H$ reflex equals $H$ max/2. Dotted lines: limits of the standard deviation.

able. The conditioning in this case includes, besides the stimulating burst, additional afferent impulses which follow the muscle contraction. Under these conditions, the excitability curve 1B differs from that illustrated in Fig. $1 \mathrm{~A}$.

In Fig. 1B, also obtained from seven subjects, the short inhibition phase of Fig. $1 \mathrm{~A}$ is not found; facilitation of the quadriceps tendon reflex is less marked and reaches only $70 \%$ of the reference values. Furthermore, the return to reference values is not regular: a second excitatory phase occurs at the interval of about $400 \mathrm{~ms}$. The reference values are reached after $600 \mathrm{~ms}$.

The standard deviations fluctuate between
20 and 30 , excluding the intervals of 40 and $180 \mathrm{~ms}$, and again 300 and $500 \mathrm{~ms}$, where they reach values of 50 or more.

Conditioning of short biceps myotatic reflex arc by activation of soleus myotatic reflex arc Activating the soleus myotatic reflex arc by the $H$ reflex leads to an inhibition of the short biceps tendon reflex which results from a decrease in excitability of the short biceps myotatic reflex arc. Both intensities of the conditioning reflex produce this effect but the shape of the curve is different.

Figure 2A, obtained from six subjects, shows that the liminal $H$ reflex inhibits the short biceps reflex. This inhibition reaches 


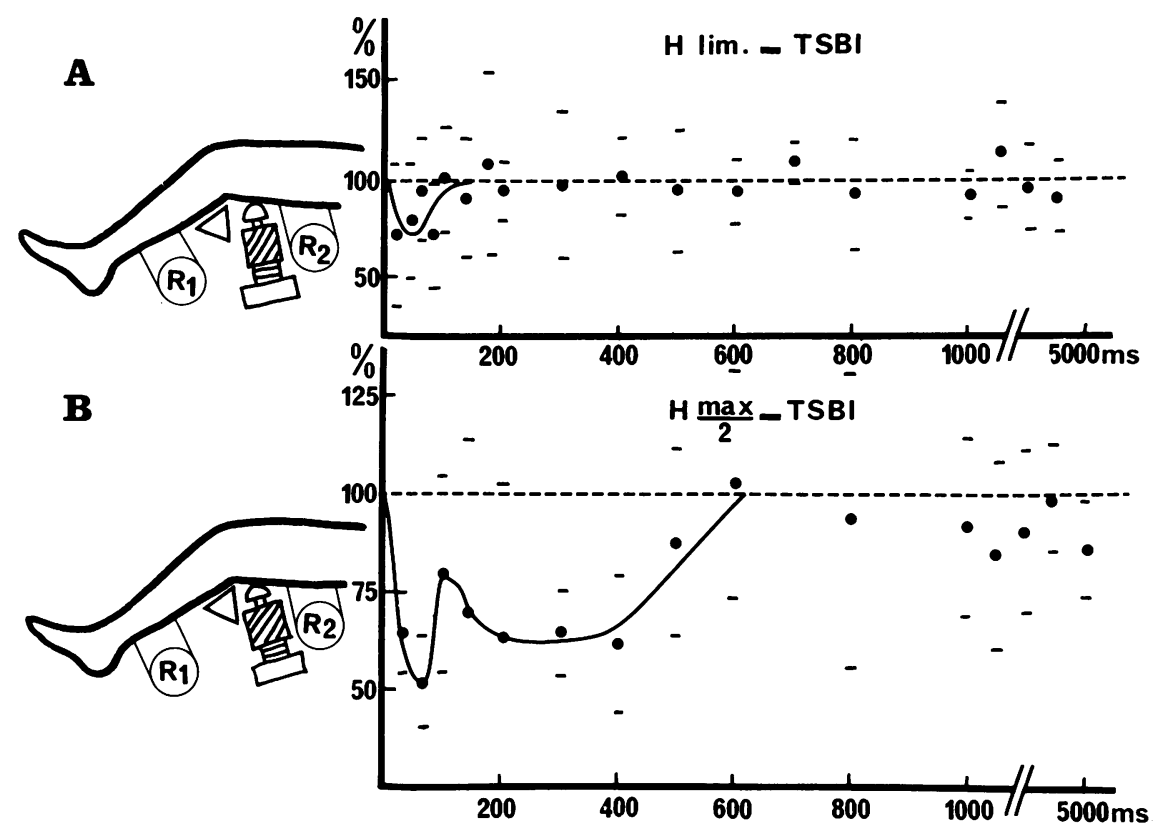

FIG. 2 Conditioning of the short biceps myotatic reflex arc by the $H$ reflex. Left: schematic representation of the experimental procedure. Symbols are the same as in Fig. 1. Right: ordinate-amplitude of the short biceps reflex expressed as a percentage of the control amplitude; abscissa-time interval in $m s$ between conditioning and test responses. In $A$, the conditioning $H$ reflex is at a liminal intensity. In $B$, the conditioning $H$ reflex equals $H$ max/2. Dotted lines: limits of the standard deviations.

$30 \%$ of reference values: after a $40 \mathrm{~ms}$ interval, it smoothly decreases and disappears after $90 \mathrm{~ms}$.

In spite of technical difficulties in eliciting the short biceps tendon reflex, the standard deviations from $180 \mathrm{~ms}$ onwards are smaller than for the quadriceps reflex (see Fig. 1): they fluctuate between 15 and 25 . Up to 180 $\mathrm{ms}$, they reach 25 to 45 .

Figure 2B illustrates the excitability curve of the short biceps reflex when conditioned by an $\mathrm{H}$ reflex corresponding to $\mathrm{H} \max / 2$.

In this case, the inhibition also reaches its maximum after $40 \mathrm{~ms}$, but it is more marked than in Fig. 2A; the decrease of this inhibition is not regular: it is first rapid up to $90 \mathrm{~ms}$, then it is slower, to be complete at $600 \mathrm{~ms}$. The standard deviations are more important
(40 to 50 ) at the intervals of 120 to $250 \mathrm{~ms}$. Otherwise, they fluctuate between 15 and 25 .

CONDITIONING OF DISTAL MYOTATIC REFLEX ARC BY ACTIVATION OF PROXIMAL MYOTATIC REFLEX ARC Conditioning of soleus myotatic reflex arc by the activation of quadriceps myotatic reflex arc The activation of the quadriceps myotatic reflex arc by its tendon reflex essentially leads to an inhibition of the soleus tendon reflex. These modifications in the excitability of the soleus arc are illustrated in Fig. 3A. This graph (obtained from seven experiments) shows that, apart from a short initial facilitatory phase (from 0 to $50 \mathrm{~ms}$ ), there is a $40 \%$ reduction of the excitability level of the soleus myotatic reflex arc compared with the reference values. 


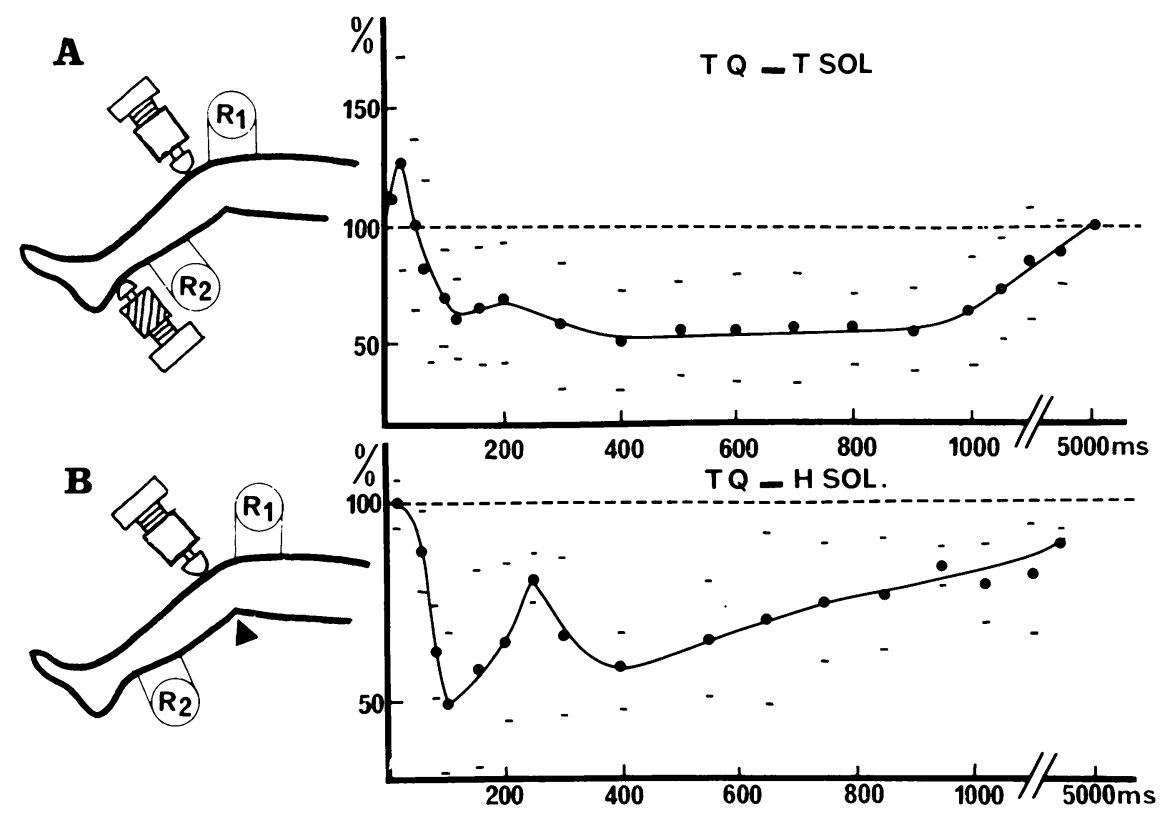

FIG. 3 Conditioning of the soleus myotatic reflex arc by a quadriceps tendon reflex. Left: schematic representation of the experimental procedure. Right: ordinate-amplitude of the soleus tendon reflex expressed as a percentage of the control amplitude; abscissa-time interval in milliseconds between conditioning and test responses. Dotted lines: limits of the standard deviations.

This inhibition is maintained at a fairly uniform level for over $1000 \mathrm{~ms}$; at the interval of $250 \mathrm{~ms}$, however, there is a slight reduction in this inhibition. From 900 to $5000 \mathrm{~ms}$ there is a progressive return to reference values.

The standard deviations fluctuate around 20 , but during the first $80 \mathrm{~ms}$ they reach values of 25 to 45 .

We also used the $H$ reflex as a test of the soleus myotatic reflex arc after conditioning by percussion of the patellar tendon (Fig. 3B).

A long-lasting inhibition is also obtained, but the rebound in excitability after an interval of about $250 \mathrm{~ms}$ is more clearly marked than in Fig. 3A. The standard deviations are generally calculated between 15 and 20; however, at intervals of 250,450 , and $1000 \mathrm{~ms}$ they are very much reduced $(\sigma=5$ to 10$)$.

Conditioning of soleus myotatic reflex arc by activation of short biceps myotatic reflex arc The excitability of the soleus myotatic reflex arc was studied in 10 subjects during the 5000 $m s$ after the activation of the short biceps arc by percussion of its tendon (Fig. 4). As in Fig. 3A, a short $50 \mathrm{~ms}$ facilitatory phase precedes a 5000-ms inhibitory phase during which the soleus tendon reflex is reduced by $30 \%$ compared with the reference values. The curve in Fig. 4 has the same general shape as Fig. 3A, but the inhibitory phase begins a little later; the disinhibition at the $250 \mathrm{~ms}$ interval is not so obvious and the return to reference values is more gradual.

The standard deviations usually fluctuate between 20 and 25 but they increase to 30 to 35 during the first $50 \mathrm{~ms}$ after the conditioning reflex, and also at intervals between 100 and $160 \mathrm{~ms}$. The similarity between curves $3 \mathrm{~A}$ and 4 could indicate that the tendon percussions of the conditioning stimuli simultaneously stimulate the antagonist muscles of the thighnamely, the quadriceps and short biceps muscles. 


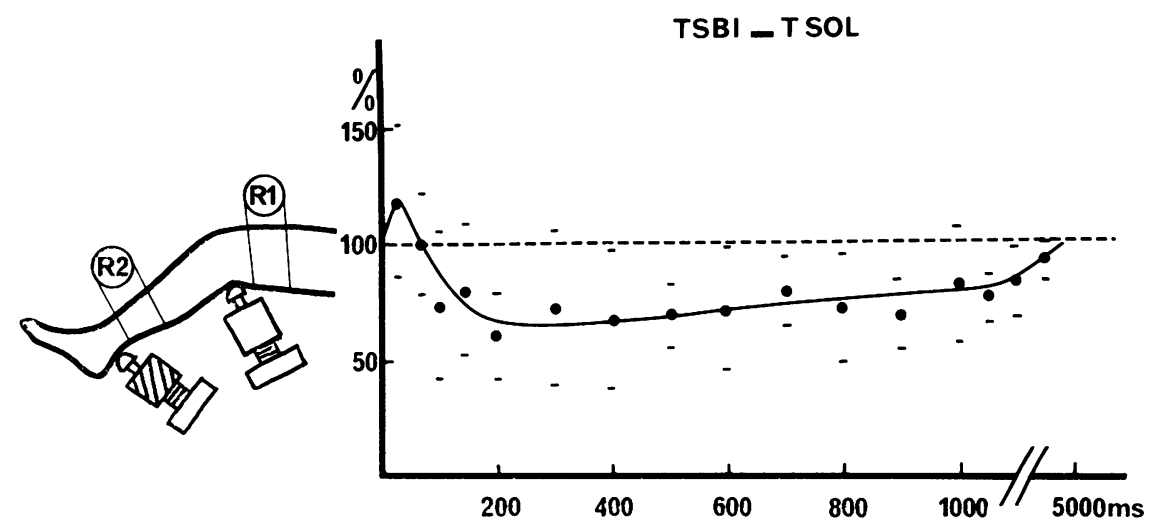

FIG. 4 Conditioning of the soleus myotatic reflex arc by a short biceps tendon reflex. Ordinate: amplitude of the soleus tendon reflex in percent of its control values. Abscissa: interval in ms between the conditioning and the test responses. Dotted lines: limits of the standard deviations.

In reply to this objection, in all experiments the activity of the quadriceps and short biceps muscles was monitored simultaneously. The percussion of the quadriceps sometimes gave rise to a response in the short biceps muscle; however, the reverse was not true: the percussion of the short biceps tendon did not elicit any electromyographical activity in the quadriceps muscle. There was, however, a subliminal activity which is illustrated in the TSBi-TQ excitability curve (Fig. 5).

Figure 5 shows that, after the short biceps myotatic reflex arc has been activated by percussing its tendon, there is an initial facilita-

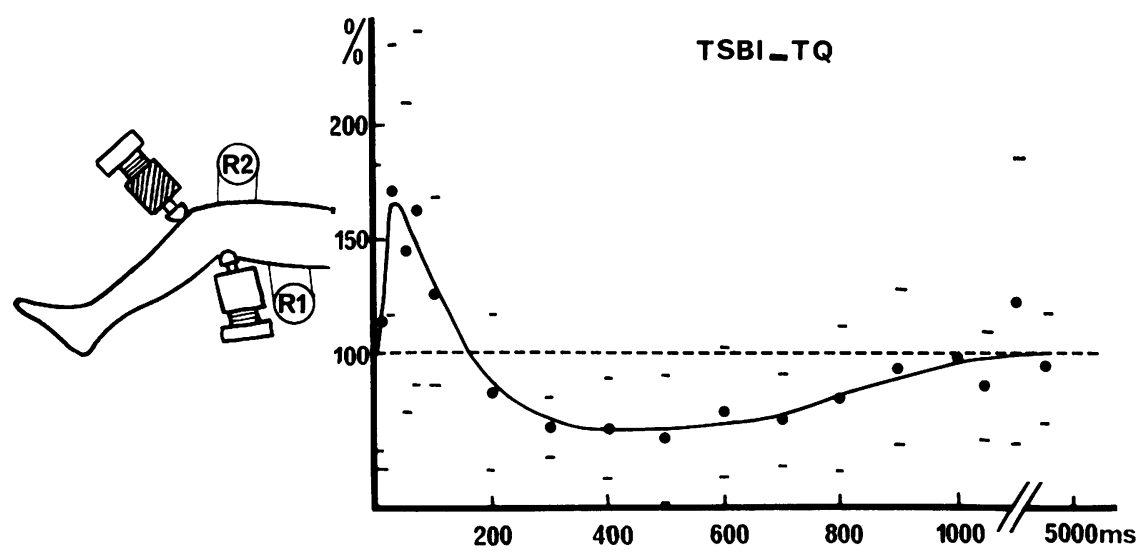

FIG. 5 Conditioning of the quadriceps myotatic reflex arc by a short biceps tendon reflex. Left: schematic representation of the experimental procedure; ordinate-amplitude of the quadriceps reflex as a percentage of its control values; abscissa-interval in $\mathrm{ms}$ between the two responses. Dotted lines: limits of the standard deviations. 
tion of the quadriceps tendon reflex; this facilitation arises very rapidly and reaches $80 \%$ of the reference values at the $40 \mathrm{~ms}$ interval, declining to zero at $120 \mathrm{~ms}$. It is subsequently replaced by an inhibition to $35 \%$ of the reference values. The reference values are progressively regained by $1000 \mathrm{~ms}$.

Another objection could be that the conditioning percussions around the knee would simultaneously stimulate the spindles of the conditioned triceps surae itself. To exclude this possibility, the mechanical activation of the quadriceps myotatic reflex arc was replaced by its electrical stimulation. This technique is less common than the electrical activation of the soleus monosynaptic arc but is nevertheless easy and adequate. We used two different conditioning intensities for the quadriceps activation: the first elicited a limi- nal electrically induced reflex; the second, the half maximum value of this response. Figure 6 shows the results. Even after a low intensity stimulation of the crural nerve, which certainly does not cause any stretch in the posterior compartment of the leg, there is a long-lasting inhibition of the Hoffmann reflex elicited in the soleus (Fig. 6A). The inhibitory effect is reinforced when the conditioning stimulation is stronger (Fig. 6B). In this latter case, the results mimic those shown in Fig. 3.

\section{DISCUSSION}

The technique of paired stimulations is currently used in neurophysiology to determine the excitability states that follow a first stimulation. The test reflex investigates the cxcitability not only of the motor neurone pool but

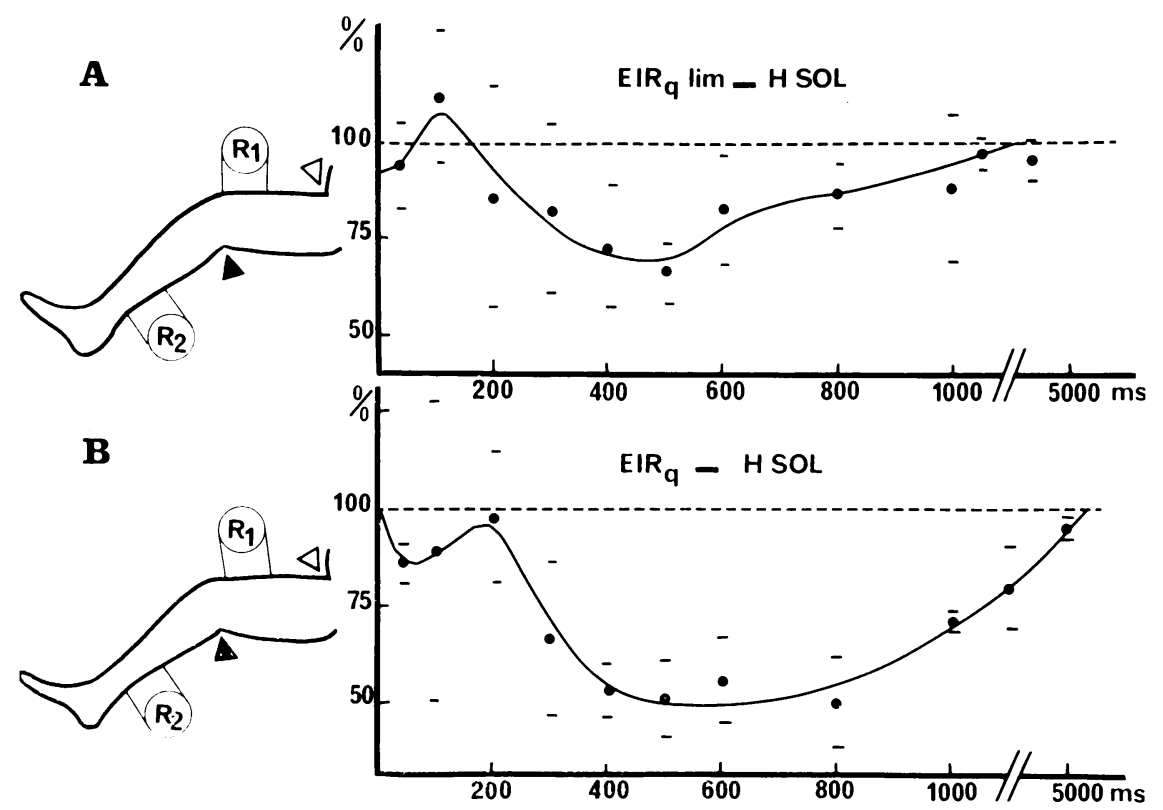

FIG. 6 Conditioning of the soleus myotatic reflex arc by a quadriceps electrically induced reflex. Left: schematic representation of the experimental procedure; ordinate-amplitude of the soleus $H$ reflex as a percentage of its control values; abscissa-interval in $m s$ between the two responses. Dotted lines: limits of the standard deviations. Upper graph $(A)$, conditioning of the soleus $H$ reflex by a quadriceps electrically induced reflex (EIR) at liminal intensity. Lower graph $(B)$, conditioning of the soleus $H$ reflex by a quadriceps EIR equal to half its maximum value. 
of the whole myotatic reflex arc. In fact, this excitability depends not only on direct influences on the motoneurones, but also upon presynaptic inhibition which acts on Ia nerve fibres (Delwaide, 1971). The advantages of this technique are to demonstrate subliminal facilitations or inhibitions and to estimate the specific role of peripheral afferent fibres in motor activities. In clinical neurophysiology, an extensive literature covers the excitability curves of the soleus myotatic reflex arc explored by two successive Hoffmann reflexes (Hoffmann, 1924; Schenck, 1951; Magladery et al., 1952; Paillard, 1955; Tabarikova and Sax, 1969). The curves thus obtained have a complex shape and can be analysed in several distinct phases. They are clearly altered in spasticity (Teasdall et al., 1952; Pinelli and Valle, 1960; Delwaide, 1971) and also in Parkinson's disease (Yap, 1967; Zander et al., 1967).

Our study uses the methodology of excitability curves to explore the influences of the reflex activation of one myotatic reflex arc on other myotatic reflex arcs of the same leg, either agonist or antagonist. Figures 1 and 2 show that the reflex activation of the soleus muscle has different effects upon the thigh muscles. The quadriceps myotatic reflex arc, being an extensor arc like that of the soleus muscle, is distinctly facilitated even for small values of the conditioning reflex. A more intense conditioning equal to $\mathrm{H} \max / 2$ does not reinforce the initial facilitatory effect. When influenced by afferent impulses from the same soleus muscle, the short biceps myotatic reflex arc shows, on the contrary, a decrease in excitability. In this case the inhibition, which is relatively small with the liminal $H$ reflex, is reinforced by the stronger conditioning reflex. The comparison between these two curves indicates, on the one hand, opposite effects on antagonist myotatic arcs, and, on the other hand, a different evolution of the effects depending on the conditioning intensity. The different effects observed in quadriceps and short biceps myotatic reflex arcs are consistent with the rules of reciprocal innervation.

The electrical stimulation used for eliciting the $\mathrm{H}$ reflex involves only the triceps muscle.
The percussion of a tendon-for example, of the thigh muscles-makes up a more complex experimental situation: the mechanical stimulus could spread to distant muscle groups and activate their proprioceptive receptors (Lance, 1965). The selectivity of the conditioning stimulation might thus be questioned. However, by using the electrical stimulation of the quadriceps myotatic reflex arc, the simultaneous activation of the soleus spindles by percussing the patella can be excluded. Moreover, although a possible participation of the quadriceps in the conditioning of the soleus myotatic reflex arc after percussion of the tendon of the biceps cannot definitely be excluded, it should be pointed out that the clinical responses observed after percussing each tendon are clearly different, indicating that the conditioning afferent impulses are not identical, at least quantitatively.

Some striking points come out of the analy-

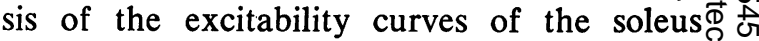
myotatic reflex arc. First, when it is condi- $\frac{\overrightarrow{\mathrm{Q}}}{\mathrm{O}}$ 을 tioned by the quadriceps reflex, inhibition occurs and the curve is quite different from $\delta$ the reciprocal curve obtained by conditioningo the quadriceps myotatic reflex arc by the $\overrightarrow{0}$ soleus reflex. Secondly, the duration of the के effects is different. It lasts longer in the case illustrated in Figs. 3A and 6, whereas in Figs. 1 and 2, and also in the excitability curves obtained by double stimulation of the soleus myotatic reflex arc (Paillard, 1955), the effect fades more rapidly. After activating the short biceps also there is a marked lasting inhibition. The excitability curves of the soleus arc do not reflect opposite effects in relation to the conditioning afferent impulses coming from either an extensor or a flexor muscle. Thus our experiments show that the proximal or distal conditioning of an extensor myotatic reflex arc have distinct effects. In the case of proximal to distal conditioning, the rules of reciprocal innervation, which seem to be true in the distal to proximal direction can no longer be applied.

There are important difficulties in the neurophysiological interpretation of the phases of the curves. First, the afferent impulses of the conditioning are complex. In the case of 
electrically induced reflexes, Ib as well as Ia afferent impulses must be considered. If the conditioning stimulation leads to a muscular response, afferent impulses will occur secondary to the muscular contraction itself. In the case of tendon reflexes, conditioning includes an initial burst in Ia afferent fibres followed by secondary afferent impulses coming from the muscular contraction. It is difficult to quantify precisely the relative contribution of these different afferent fibres to the conditioning message. Moreover, our understanding of the specific role of the various afferent fibres is becoming increasingly complex and the problems of the influences they might have in animals are not completely solved (Eccles et al., 1957; Eccles and Lundberg, 1958; Granit et al., 1957; Tsukuhara, and Ohye, 1964; Hongo et al., 1969). In man, the relationships between afferent nerve fibres and motoneurones are at the present time purely speculative.

In clinical neurophysiology it should be remembered that, in addition to the complexity of the purely spinal action of afferent fibres, the experiments are made with intact individuals and that each phase of the excitability curves can reflect the influence of supraspinal mechanisms also. Because the return to reference values in Figs. 3, 4, and 6 lasts for several seconds, it would be logical to suppose that these supraspinal mechanisms have a decisive action. However, this point cannot be taken for granted because inhibitions lasting a few seconds have been noted in patients with spinal cord transection (Shahani and Young, 1971). In fact, it is likely that an excitability curve might result from several mechanisms, some of them facilitatory and others inhibitory, which appear after different delays and with distinct durations. For a time to come, their analysis will probably remain an open question.

In order to simplify the experimental situation, we used small and liminal stimulations. The comparison of the results obtained with various intensities of conditioning is interesting. Thus a reduced facilitation of the quadriceps reflex observed $100 \mathrm{~ms}$ after a more intense stimulation of the sciatic nerve could be ascribed to the activation of nerve fibres of smaller diameter (Fig. 1,B). The use of liminal stimulus also makes it possible to appreciate the part played by further afferent impulses secondary to the muscular contraction. Comparison of Figs. 1, A and 1,B suggests that the facilitation observed at $400 \mathrm{~ms}$ (Fig. 1,B) is secondary to new afferent impulses and perhaps more precisely to Ia afferent impulses which are particularly abundant during the phase of muscular relaxation (Granit et al., 1966).

Finally, it must be pointed out that the conclusions of this study cannot necessarily be applied to voluntary as opposed to reflex contractions. During voluntary movements such as walking, interneurones are facilitated or inhibited to ensure that afferent impulses produce the desired effects within the spinal cord.

\section{REFERENCES}

Delwaide, P. J. (1971). Étude Expérimentale de l'Hyperréflexie Tendineuse en Clinique Neurologique, p. 324. Editions Arscia: Bruxelles.

Delwaide, P. J., Hugon, M., and Thiriet, P. (1970). Slow stretching of human triceps surae by a pneumatic device. Electromyography, 4, 399-405.

Desmedt, J. E. (1973). Human reflexes. Pathophysiology of motor systems. Methodology of human reflexes. In New Developments in Electromyography and Clinical Neurophysiology, vol. 3. Edited by J. E. Desmedt. Karger: Basel.

Eccles, J. C., Eccles, R. M., and Lundberg, A. (1957). Synaptic actions on motoneurones caused by impulses in Golgi tendon organ afferents. Journal of Physiology, 138, 227-252.

Eccles, R. M., and Lundberg, A. (1958). Integrative pattern of Ia synaptic actions on motoneurones of hip and knee muscles. Journal of Physiology, 144, 271-298.

Granit, R., Kellerth, J. O., and Szumski, A. J. (1966). Intracellular autcgenetic effects of muscular contraction on extensor motoneurones. The silent period. Journal of Physiology, 182, 484-503.

Granit, R., Phillips, C. G., Skoglund, S., and Steg, G. (1957). Differentiation of tonic from phasic alpha ventral horn cells by stretch pinna and crossed extensor reflexes. Journal of Neurophysiology, 20, 470-481.

Hoffmann, P. (1924). Untersuchungen über die refraktäre periode des menschlichen Rücken markes. Zeitschrift für Biologie, 81, 37-48.

Hongo, T., Jankowska, E., and Lundberg, A. (1969). The rubrospinal tract II. Facilitation of interneuro- 
nal transmission in reflex paths to motoneurones. Experimental Brain Research, 7, 365-391.

Lance, J. W. (1965). The mechanism of reflex irradiation. Proceedings of the Australian Association of Neurology, 3, 77-82.

Lloyd, D. P. (1944). Functional organization of the spinal cord. Physiological Review, 24, 1-17.

Magladery, J. W., Teasdall, R. D., Park, A. M., and Languth, H. W. (1952). Electrophysiological studies on reflex activity in patients with lesions of the nervous system. I. A comparison of spinal motoneurone excitability following afferent nerve volleys in normal persons and patients with upper motor neurone lesions. Bulletin of the Johns Hopkins Hospital, 91, 219-244.

Magladery, J. W., Teasdall, R. D., Park, A. M., and Porter, W. E. (1951). Electrophysiological studies of nerve and reflex activity in normal man. V. Excitation and inhibition of two-neurone reflexes by afferent impulses in the same nerve trunk. Bulletin of the Johns Hopkins Hospital, 88, 520-537.

Paillard, J. (1955). Réflexes et Régulations d'Origine Proprioceptive chez l'Homme, p. 293. Arnette: Paris.

Pinelli, P., and Valle, M. (1960). Studio fisiopatologico dei riflessi muscolari nelle paresi spatische (sui tests per la misura della spasticita). Archivio Scienze Mediche, 109, 1-127.
Schenck, E. (1951). Untersuchungen über die Hemmungsphase nach einen Zweineurone (Eigen) reflex beim Menschen. Pflügers Archiv für die gesamte Physiologie, 153, 286-300.

Shahani, B. T., and Young, R. R. (1971). Human flexor reflexes. Journal of Neurology, Neurosurgery and Psychiatry, 34, 616-627.

Tabarikova, H., and Sax, D. S. (1969). Conditioning of H-reflex by a preceding subthreshold H-reflex stimulus. Brain, 92, 203-212.

Teasdall, R. D., Park, A. M., Languth, H. W., and Magladery, J. W. (1952). Electrophysiological studies of reflex activity in patients with lesions of the nervous system. Disclosure of normally suppressed monosynaptic reflex discharge of spinal motoneurones by lesions of lower brain-stem and spinal cord. Bulletin of the Johns Hopkins Hospital, 91, 245-256.

Tsukuhara, N., and Ohye, C. (1964). Polysynaptic activation of extensor motoneurons from group Ia fibres in cat spinal cord. Experientia, 20, 628-629.

Yap, C. B. (1967). Spinal segmental and long-loop reflexes on spinal motoneurone excitability in spasticity and rigidity. Brain, 90, 887-896.

Zander Olsen, P., and Diamantopoulos, E. (1967). Excitability of spinal motoneurones in normal subjects and patients with spasticity, rigidity and cerebellar hypotonia. Journal of Neurology, Neurosurgery, and Psychiatry, 30, 325-331. 